\title{
Muscarinic Receptor Modulation of Basal and $\beta$-Adrenergic Stimulated Function of the Failing Human Left Ventricle
}

\author{
Gary E. Newton, ${ }^{*}$ Andrea B. Parker, ${ }^{\ddagger}$ Joel S. Landzberg, ${ }^{\S}$ Wilson S. Colucci, ${ }^{\S}$ and John D. Parker ${ }^{\star}$ \\ *Cardiovascular Division, Department of Medicine, Mount Sinai Hospital, the University of Toronto, Toronto, Ontario; ${ }^{*}$ Socar Research \\ SA, Givrins, Switzerland; and ${ }^{\S}$ Cardiovascular Division, Department of Medicine, Brigham and Women's Hospital and Harvard Medical
} School, Boston, Massachusetts

\begin{abstract}
The objective of this study was to evaluate the effect of muscarinic receptor modulation on basal and $\beta$-adrenergic stimulated left ventricular function in patients with heart failure. 21 heart failure patients and 14 subjects with normal ventricular function were studied. In Protocol 1 intracoronary acetylcholine resulted in a $60 \pm 8 \%$ inhibition of the left ventricular $+\mathrm{dP} / \mathrm{dt}$ response to intracoronary dobutamine in the normal group, and a similar $70 \pm 13 \%$ inhibition in the heart failure group. Acetylcholine also attenuated the dobutamine-mediated acceleration of isovolumic relaxation (Tau) in both groups. Acetylcholine alone had no effect on Tau in the normal group, while it prolonged Tau in the heart failure group. In Protocol 2 intracoronary atropine resulted in a $35 \pm 10 \%$ augmentation of the inotropic response to dobutamine in the normal group, versus a non-significant $12 \pm 15 \%$ augmentation of the dobutamine response in the heart failure group. In Protocol 3, in 6 heart failure patients, both effects of acetylcholine, the slowing of ventricular relaxation and the inhibition of $\beta$-adrenergic responses, were reversed by the addition of atropine. Therefore, in the failing human left ventricle muscarinic stimulation has an independent negative lusitropic effect and antagonizes the effects of $\beta$-adrenergic stimulation. (J. Clin. Invest. 1996. 98:2756-2763.) Key words: acetylcholine • atropine • diastole $\cdot$ dobutamine $\cdot$ heart failure, congestive
\end{abstract}

\section{Introduction}

Parasympathetic outflow to the heart is reduced in patients with heart failure $(1,2)$. The importance of this abnormality in the pathogenesis of life-threatening rhythm disturbances has been observed (3), and the possible utility of pharmacological augmentation of parasympathetic tone in patients with heart failure has been suggested (4). However, the effect of myocardial muscarinic receptor modulation on ventricular function in the setting of heart failure is poorly understood. This question is important because stimulation of myocardial muscarinic re-

Address correspondence to John D. Parker, Cardiovascular Division, Room 1609, Mount Sinai Hospital, 600 University Ave., Toronto, Ontario, M5G 1X5. Phone: 416-586-4794; FAX: 416-586-8413.

Received for publication 23 October 1995 and accepted in revised form 8 October 1996.

J. Clin. Invest.

(C) The American Society for Clinical Investigation, Inc. 0021-9738/96/12/2756/08 \$2.00

Volume 98, Number 12, December 1996, 2756-2763 ceptors has potential negative effects on contractile performance (5) and can attenuate $\beta$-adrenergic receptor mediated increases in contractility (6-9).

In various animal models of heart failure muscarinic receptor density in ventricular myocardium has been reported to be either reduced $(10,11)$ or increased $(5,12)$, while receptor density in cardiac tissue from patients with heart failure has been shown to be unchanged (13-15). Muscarinic receptors couple to the inhibitory $G$ protein, $G_{i}$, which has increased activity in the failing heart $(5,15-18)$. Vatner et al. (5) have recently confirmed that heart failure, induced by rapid ventricular pacing in dogs, is associated with an increase in muscarinic receptor density and $\mathrm{G}_{\mathrm{i}}$ in ventricular myocardium. The functional significance of this receptor pathway upregulation was also demonstrated in that muscarinic stimulation in animals with heart failure resulted in greater inhibition of adenylate cyclase activity and myocardial contractility than was observed in normal animals. This important study confirms that muscarinic receptor pathways are at least intact and appear to be upregulated in the setting of heart failure. Of note, muscarinic stimulation can also modify ventricular performance through its effects on $\beta$-adrenergic receptor-mediated responses. Previous studies in normal animals have demonstrated that vagal stimulation attenuates $\beta$-adrenergic-mediated increases in contractility (6-8). Similarly, in humans with normal ventricular function, we have demonstrated that muscarinic stimulation attenuates $\beta$-adrenergic-mediated inotropic responses, while having no effect on basal contractility (9). The effect of muscarinic stimulation on ventricular diastolic function has not been studied in humans. In animals with normal ventricular function muscarinic stimulation has been reported to either slow $(7,19)$ or have no effect (20) on ventricular relaxation.

The effect of muscarinic stimulation and blockade on left ventricular function has not been described in the setting of human congestive heart failure. Given normal receptor density (13-15), increased $\mathrm{G}_{\mathrm{i}}$ activity $(5,15-18)$, and the negative inotropic effects of muscarinic stimulation in an animal model of heart failure (5), muscarinic receptor modulation may have important effects on ventricular function in human congestive heart failure. Therefore, this study was performed to determine the effect of myocardial muscarinic receptor modulation on basal and dobutamine stimulated left ventricular systolic and diastolic function in patients with heart failure, and to contrast these effects with observations made in subjects with normal ventricular function.

\section{Methods}

Study population. The study population consisted of 35 subjects referred for diagnostic catheterization. The subjects in the normal ventricular function group ( $n=14 ; 7$ males and 7 females) were free of significant coronary artery stenosis by angiography, had normal baseline hemodynamics and a normal left ventricular ejection fraction. 
4 subjects with normal ventricular function were included in a previous study of the effects of muscarinic pathways on left ventricular contractile function (9). These subjects were included in the present study because they had undergone an identical protocol and had left ventricular pressure recordings that allowed for calculation of the rate of ventricular relaxation (which was neither analyzed or reported in the previous study). Medical treatment in the normal group, for either hypertension or a chest pain syndrome, consisted of calcium channel blockers ( 3 subjects), $\beta$-blockers ( 4 subjects), and angiotensin-converting enzyme inhibitors (3 subjects). These medications were withheld for at least 24 hours before this investigation.

The patients with heart failure ( $n=21 ; 15$ males and 6 females) all suffered from dilated cardiomyopathy with normal coronary arteries. 1 heart failure patient was in atrial fibrillation and the heart rate responses from this patient were not included in the analysis. The etiology of dilated cardiomyopathy was idiopathic in 16 patients, ethanol related in 4 patients, and chemotherapy in 1 patient. All had significant left ventricular systolic dysfunction. Medical therapy consisted of furosemide, (18 patients), digoxin (13 patients), angiotensinconverting enzyme inhibitors (17 patients), and nitrates (3 patients). In the heart failure group medical therapy was withheld on the morning of the investigation.

The investigation was begun at the Brigham and Women's Hospital (Boston, MA) and completed at the Mount Sinai Hospital (Toronto, Ontario). At both centers the protocol was approved by the ethical review committee for experimentation involving human subjects. Written informed consent was obtained in all cases.

Hemodynamic measurements. Following a diagnostic heart catheterization $20 \mathrm{~min}$ elapsed before beginning this investigation. A 7Fr micromanometer-tipped catheter (Millar Industries, Houston, TX) was placed in the left ventricle. A 7Fr left Judkins catheter (Cordis Laboratories, Miami, FL), placed from the opposite femoral artery, was advanced to the ostium of the left main coronary artery for intracoronary drug infusions. Femoral artery pressure was monitored via an 8Fr side-arm sheath (Cordis Laboratories, Miami, FL). The electrocardiogram, left ventricular pressure, and its first derivative $(\mathrm{dP} / \mathrm{dt}$, continuous electronic differentiation) were recorded on a strip chart recorder at a paper speed of $100 \mathrm{~mm} / \mathrm{s}$. Measurements for heart rate, left ventricular peak $+\mathrm{dP} / \mathrm{dt}$ and peak $-\mathrm{dP} / \mathrm{dt}$, and left ventricular systolic and end-diastolic pressure were made by averaging at least 15 beats under each experimental condition.

Experimental approach. The effect of muscarinic receptor stimulation and blockade on basal and dobutamine stimulated left ventricular function was assessed in three drug infusion protocols. In all parts of this experiment intracoronary drugs were administered into the left main coronary artery via the Judkins catheter using a Harvard pump (South Natick, MA) for 4-5 min with hemodynamic measurements made in the final minute. After completion of drug infusions, radiographic contrast was injected to confirm the continued position of the catheter in the left main coronary ostium.

Protocol 1. Intracoronary dobutamine and acetylcholine. In this protocol the effect of acetylcholine on dobutamine-mediated increases in systolic and diastolic left ventricular function was assessed. 10 subjects with normal ventricular function and 11 patients with heart failure were studied. The sequence of infusions was as follows: $(a)$ Control $5 \%$ dextrose in water $\left(\mathrm{D}_{5} \mathrm{~W}\right)$, the vehicle for intracoronary drug infusion, at $2 \mathrm{ml} / \mathrm{min}$. (b) Intracoronary dobutamine with upward titration $(12.5,25,37.5$, and $50 \mu \mathrm{g} / \mathrm{min})$ until at least a $25 \%$ increase in $+\mathrm{dP} / \mathrm{dt}$ was achieved, or until the patient experienced tachycardia or increasing extrasystoles. The mean dobutamine infusion rate was $19 \pm 4 \mu \mathrm{g} / \mathrm{min}$ in the normal ventricular function group, and $23 \pm 2 \mu \mathrm{g} /$ min in the heart failure group. (c) Recontrol $\mathrm{D}_{5} \mathrm{~W}$ until $+\mathrm{dP} / \mathrm{dt}$ had returned to within $10 \%$ of its control value. (d) Intracoronary acetylcholine, at concentrations of $10^{-6}, 10^{-5}$, and $10^{-4} \mathrm{~mol} / \mathrm{L}$ consecutively, at $1.25 \mathrm{ml} / \mathrm{min}$ to achieve estimated intracoronary concentrations of $10^{-8}, 10^{-7}$, and $10^{-6} \mathrm{~mol} / \mathrm{L}$, assuming left main coronary blood flow of $125 \mathrm{ml} / \mathrm{min}$. (e) Simultaneous infusions of acetylcholine and dobutamine at the same infusion rates as were used initially.
Protocol 2. Intracoronary dobutamine and atropine. In this protocol, the effect of muscarinic receptor blockade on dobutamine responses was assessed. Infusions were identical to those in Protocol 1, except that intracoronary atropine $(12 \mu \mathrm{g} / \mathrm{min})$ was substituted for acetylcholine. The mean dobutamine infusion rate was $16 \pm 3 \mu \mathrm{g} / \mathrm{min}$ in the normal ventricular function group and $24 \pm 1 \mu \mathrm{g} / \mathrm{min}$ in the heart failure group. In total 7 subjects with normal ventricular function and 9 heart failure patients were studied. This included 3 subjects with normal ventricular function and 5 heart failure patients who participated in Protocol 1. In the latter group the order of intracoronary drug infusions was $(a)$ Control $\mathrm{D}_{5} \mathrm{~W},(b)$ dobutamine, $(c)$ recontrol $\mathrm{D}_{5} \mathrm{~W},(d)$ acetylcholine, $(e)$ acetylcholine and dobutamine, $(f)$ second recontrol $\mathrm{D}_{5} \mathrm{~W},(g)$ atropine, and finally, $(h)$ atropine and dobutamine. In this group of patients the second recontrol was used as the recontrol period for Protocol 2. Of note, there were no changes in $+\mathrm{dP} / \mathrm{dt}$ when the initial control period and the second recontrol period before the infusion of atropine were compared in these patients.

Protocol 3. Intravenous dobutamine and combined intracoronary acetylcholine and atropine. Six heart failure patients were studied in this protocol which was designed to explore whether the acetylcholine responses could be reversed by a concurrent infusion of atropine. This would confirm that the acetylcholine responses resulted from muscarinic receptor stimulation, and that the dose of atropine used in Protocol 2 was sufficient to antagonize the effects of a muscarinic receptor agonist in the setting of heart failure. An additional rationale for Protocol 3 was to demonstrate that the modulation of the dobutamine response by acetylcholine was not simply the result of altered coronary blood flow with resultant changes in the intracoronary concentration of dobutamine. This was accomplished by using an intravenous infusion of dobutamine (mean infusion rate $5.8 \pm 0.5 \mu \mathrm{g} / \mathrm{kg} / \mathrm{min}$ ). In this protocol the sequence of drug infusions was, $(a)$ Control intracoronary $\mathrm{D}_{5} \mathrm{~W},(b)$ intravenous dobutamine to achieve at least a $25 \%$ increase in $+\mathrm{dP} / \mathrm{dt},(c)$ recontrol $\mathrm{D}_{5} \mathrm{~W},(d)$ intracoronary acetylcholine $\left(10^{-6}\right.$ to $\left.10^{-4} \mathrm{~mol} / \mathrm{L}\right),(e)$ intravenous dobutamine and intracoronary acetylcholine, $(f)$ intravenous dobutamine, plus intracoronary acetylcholine and atropine $(12 \mu \mathrm{g} / \mathrm{min})$, and finally, $(\mathrm{g})$ acetylcholine and atropine.

Left ventricular isovolumic relaxation. The time constant of left ventricular relaxation, Tau, was calculated in two different ways. The first method is a modification of that described by Weiss et al., such that Tau $\left(T_{L}\right)=-1$ slope of the regression line for the natural logarithm of left ventricular pressure versus time for the period from peak $-\mathrm{dP} / \mathrm{dt}$ to $5 \mathrm{~mm} \mathrm{Hg}$ above left ventricular end-diastolic pressure (21). The second method is the direct measurement of the pressure halftime $\left(\mathrm{T}_{1 / 2}\right)$, as described by Mirsky (22). With this method, Tau is directly measured from the pressure tracing as the time required for left ventricular pressure to fall to one-half of its value at peak $-\mathrm{dP} / \mathrm{dt}$. In Protocol 1 and 2 left ventricular pressure recordings were digitized by hand at 2- to 4-ms intervals using a digitizing tablet (Summagraphics, Summagraphics Corporation, Fairfield, CT) interfaced with a Macintosh personal computer. In Protocol 3, left ventricular pressure and the electrocardiogram were digitally recorded at $300 \mathrm{~Hz}$ using a Macintosh personal computer equipped with a multichannel analogue to digital converter. Data files were stored to disc for later analysis. Using customized software developed in Labview (Version 3.0; National Instruments Corporation, Austin, TX) Tau was calculated off-line using identical methods to those described above. In all cases values for Tau represent the mean calculated from 8 cardiac cycles during each experimental condition.

The reproducibility of the manual method for calculation of Tau was assessed according to the method of Bland and Altman (23). Both $T_{L}$ and $T_{1 / 2}$ were measured from pressure tracings of 14 patients in duplicate by the same observer on two occasions at least $2 \mathrm{~d}$ apart. For $\mathrm{T}_{\mathrm{L}}$, the mean of the differences of 14 pairs of measurements was $0.41 \pm 1.95 \mathrm{~ms}$ (mean \pm standard deviation). Therefore, the coefficient of repeatability (two standard deviations) for $\mathrm{T}_{\mathrm{L}}$ is $3.90 \mathrm{~ms}$. For $\mathrm{T}_{1 / 2}$, the mean of the differences was $0.08 \pm 1.47 \mathrm{~ms}$. Therefore, the coefficient of repeatability for $\mathrm{T}_{1 / 2}$ is $2.94 \mathrm{~ms}$. 
Table I. Baseline Characteristics

\begin{tabular}{lcc}
\hline & Normal LV function & Heart failure \\
\hline \multicolumn{1}{c}{$n$} & 14 & 21 \\
Age $(\mathrm{yr})$ & $54 \pm 3$ & $54 \pm 2$ \\
$\mathrm{LVEF}(\%)$ & $67 \pm 5$ & $26 \pm 3^{*}$ \\
$\mathrm{HR}(\mathrm{bpm})$ & $76 \pm 4$ & $90 \pm 4^{*}$ \\
$\mathrm{Mean}$ PAP $(\mathrm{mmHg})$ & $12 \pm 1$ & $26 \pm 3^{*}$ \\
$\mathrm{LVSP}(\mathrm{mmHg})$ & $124 \pm 5$ & $114 \pm 5$ \\
$\mathrm{LVEDP}(\mathrm{mmHg})$ & $10 \pm 1$ & $20 \pm 2^{*}$ \\
$+\mathrm{dP} / \mathrm{dt}(\mathrm{mmHg} / \mathrm{s})$ & $1487 \pm 77$ & $869 \pm 70^{*}$ \\
$\mathrm{~T}_{\mathrm{L}}(\mathrm{ms})$ & $41 \pm 2$ & $56 \pm 3^{*}$ \\
$\mathrm{~T}_{1 / 2}(\mathrm{~ms})$ & $30 \pm 2$ & $39 \pm 2^{*}$ \\
\end{tabular}

LVEF, left ventricular ejection fraction; HR, heart rate; mean PAP, mean pulmonary artery pressure; LVSP, left ventricular systolic pressure; LVEDP, left ventricular end-diastolic pressure; $+\mathrm{dP} / \mathrm{dt}$, peak positive left ventricular $\mathrm{dP} / \mathrm{dt} ; \mathrm{T}_{\mathrm{L}}$, Tau, logarithmic method; $\mathrm{T}_{1 / 2}$, Tau, direct pressure half-time method. $* P<0.05$ versus normal left ventricular (LV) function group.

Statistical analysis. All data sets were tested for normality by means of the Shapiro-Wilk test and found to be normally distributed. Baseline characteristics were compared using unpaired two-tailed $t$ tests. For Protocols 1 and 2 comparisons of the means at each experimental step between groups were done using repeated measures MANOVA, with appropriate contrast statements and adjusting for multiple comparisons. Comparisons within groups were done in the same manner but fitting the model without the intercept. For Protocol 3 a repeated measures MANOVA was used to compare the means at each experimental step, contrasting the comparison of interest and adjusting for multiple comparisons. All data are presented as mean \pm SEM. Statistical analysis was performed in SAS (release 6.10; SAS Institute Inc., Cary, NC).

Table II. Responses to Dobutamine, Acetylcholine, and Their Combination (Protocol 1)

\begin{tabular}{|c|c|c|c|c|c|}
\hline & $\mathrm{C}$ & $\Delta$ Dob & $\mathrm{RC}$ & $\Delta$ Ach & $\Delta \mathrm{Dob}+\mathrm{Ach}$ \\
\hline \multicolumn{6}{|l|}{ Normal } \\
\hline HR & $81 \pm 4$ & $+2 \pm 2$ & $78 \pm 4$ & $-1 \pm 2$ & $+1 \pm 3$ \\
\hline LVEDP & $11 \pm 2$ & $-3 \pm 1 *$ & $10 \pm 1$ & $0 \pm 1$ & $0 \pm 1$ \\
\hline LVSP & $124 \pm 6$ & $+13 \pm 2 *$ & $126 \pm 6$ & $-1 \pm 3$ & $+7 \pm 5$ \\
\hline$+\mathrm{dP} / \mathrm{dt}$ & $1502 \pm 104$ & $+631 \pm 98^{*}$ & $1503 \pm 103$ & $+8 \pm 31$ & $+221 \pm 48$ \\
\hline $\mathrm{T}_{\mathrm{L}}$ & $40 \pm 3$ & $-6 \pm 2 *$ & $39 \pm 3$ & $-1 \pm 1$ & $-1 \pm 2^{\ddagger}$ \\
\hline $\mathrm{T}_{1 / 2}$ & $28 \pm 2$ & $-4 \pm 2 *$ & $28 \pm 2$ & $+1 \pm 1$ & $0 \pm 1$ \\
\hline \multicolumn{6}{|l|}{$\mathrm{CHF}$} \\
\hline HR & $89 \pm 4$ & $-1 \pm 2$ & $87 \pm 5$ & $+2 \pm 1$ & $+3 \pm 2$ \\
\hline LVEDP & $20 \pm 2$ & $-2 \pm 1$ & $20 \pm 2$ & $+3 \pm 1 * \S$ & $+2 \pm 1$ \\
\hline LVSP & $122 \pm 9$ & $+5 \pm 3 * \S$ & $124 \pm 9$ & $+2 \pm 2$ & $+4 \pm 4$ \\
\hline$+\mathrm{dP} / \mathrm{dt}$ & $1035 \pm 101$ & $+261 \pm 37 * \S$ & $1072 \pm 105$ & $-19 \pm 14$ & $+81 \pm 30 * \neq \S$ \\
\hline $\mathrm{T}_{\mathrm{L}}$ & $51 \pm 3$ & $-7 \pm 1^{*}$ & $52 \pm 3$ & $+6 \pm 1 * \S$ & $0 \pm 1^{\ddagger}$ \\
\hline $\mathrm{T}_{1 / 2}$ & $34 \pm 2$ & $-4 \pm 1 *$ & $34 \pm 1$ & $+3 \pm 1 *$ & $-2 \pm 1$ \\
\hline
\end{tabular}

Normal, normal ventricular function group; CHF, congestive heart failure group; $\mathrm{C}$ and $\mathrm{RC}$, absolute values at control and recontrol; $\Delta \mathrm{Dob}$, $\Delta \mathrm{Ach}$, ahd $\Delta \mathrm{Dob}+\mathrm{Ach}$, change from preceding control period in response to dobutamine, acetylcholine, and their combination. See Table I for remainder of abbreviations. ${ }^{*} P<0.05$ for within group responses, ${ }^{\S} P<0.05$ versus response in the normal ventricular function group, ${ }^{\ddagger} P<$ 0.05 for comparison of dobutamine plus acetylcholine response versus dobutamine response.

\section{Results}

Baseline hemodynamics. Baseline hemodynamics and indices of left ventricular contractility and isovolumic relaxation (Tau) for all subjects with normal ventricular function, and all patients with heart failure are summarized in Table I. The correlation coefficients for the linear regression of the logarithm of left ventricular pressure versus time during isovolumic relaxation was $0.992 \pm 0.001$ in the normal ventricular function group, and $0.991 \pm 0.001$ in the heart failure group.

Protocol 1. Intracoronary dobutamine and acetylcholine. Intracoronary dobutamine increased left ventricular systolic pressure in both groups, and reduced left ventricular end-diastolic pressure in the normal ventricular function group (Table II). There were no hemodynamic changes in the normal group in response to intracoronary acetylcholine. In contrast, in the heart failure group acetylcholine increased left ventricular end-diastolic pressure, a change which was significantly different compared to the response in the normal group. The combi-

\section{A}

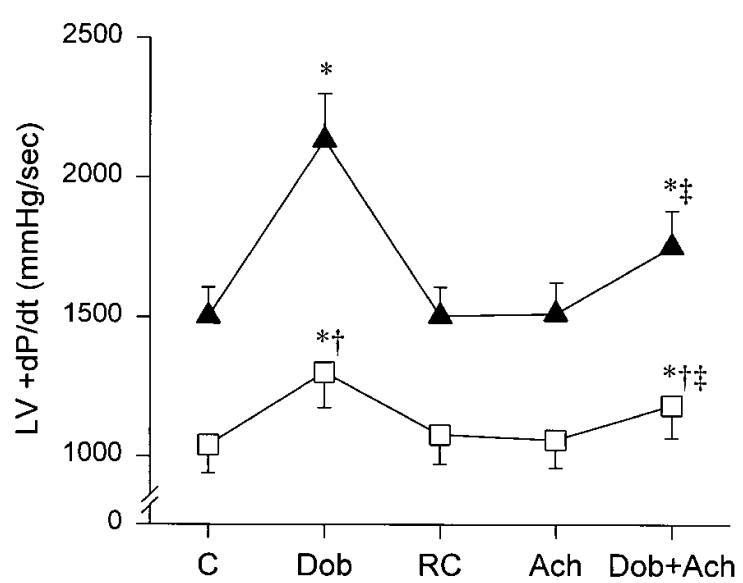

$\mathbf{B}$

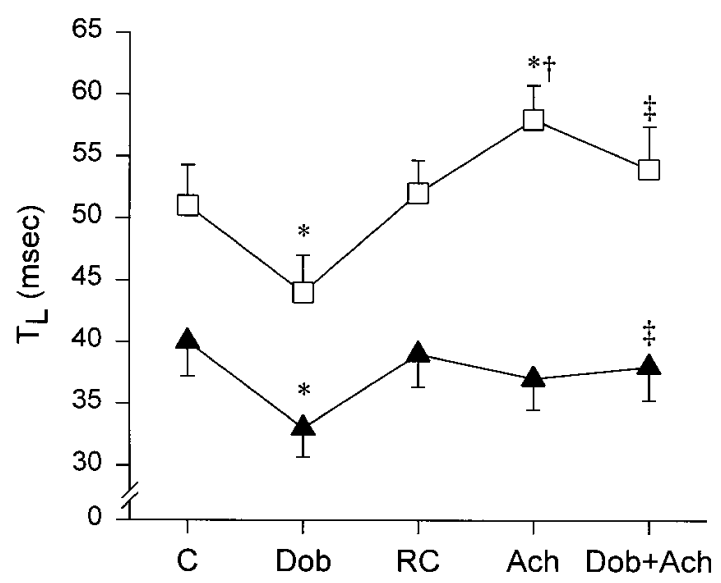

Figure 1. Left ventricular $+\mathrm{dP} / \mathrm{dt}(A)$ and $\mathrm{T}_{\mathrm{L}}(B)$ in the normal ventricular function group (solid triangles) and the heart failure group (open squares) in Protocol 1. Control $(C)$, intracoronary dobutamine $(D o b)$, recontrol $(R C)$, acetylcholine $(A c h)$. Data are presented as mean \pm SEM. ${ }^{*} P<0.05$ versus the preceding control period, ${ }^{\dagger} P<$ 0.05 versus the normal ventricular function group, ${ }^{\ddagger} P<0.05$ for the comparison of dobutamine plus acetylcholine versus dobutamine alone. 
Table III. Responses to Dobutamine, Atropine, and Their Combination (Protocol 2)

\begin{tabular}{|c|c|c|c|c|c|}
\hline & $\mathrm{C}$ & $\Delta$ Dob & $\mathrm{RC}$ & $\Delta$ Atr & $\Delta \mathrm{Dob}+$ Atr \\
\hline \multicolumn{6}{|l|}{ Normal } \\
\hline HR & $73 \pm 7$ & $+5 \pm 3$ & $76 \pm 7$ & $+5 \pm 3$ & $+14 \pm 8^{*}$ \\
\hline LVEDP & $13 \pm 2$ & $-4 \pm 1 *$ & $12 \pm 2$ & $-1 \pm 1$ & $-3 \pm 1 *$ \\
\hline LVSP & $127 \pm 9$ & $+9 \pm 2$ & $126 \pm 8$ & $+2 \pm 5$ & $+10 \pm 2$ \\
\hline$+\mathrm{dP} / \mathrm{dt}$ & $1460 \pm 140$ & $+814 \pm 104 *$ & $1474 \pm 116$ & $+107 \pm 66$ & $+1100 \pm 171$ \\
\hline $\mathrm{T}_{\mathrm{L}}$ & $46 \pm 3$ & $-6 \pm 2$ & $45 \pm 3$ & $-2 \pm 2$ & $-9 \pm 2 *$ \\
\hline $\mathrm{T}_{1 / 2}$ & $35 \pm 3$ & $-4 \pm 2$ & $33 \pm 3$ & $-1 \pm 1$ & $-7 \pm 1 *$ \\
\hline \multicolumn{6}{|l|}{$\mathrm{CHF}$} \\
\hline HR & $95 \pm 6$ & $-2 \pm 5$ & $93 \pm 7$ & $+1 \pm 1$ & $+4 \pm 4$ \\
\hline LVEDP & $20 \pm 3$ & $-1 \pm 1$ & $21 \pm 2$ & $+1 \pm 1$ & $-3 \pm 2$ \\
\hline LVSP & $109 \pm 9$ & $+6 \pm 1$ & $112 \pm 9$ & $0 \pm 1$ & $+3 \pm 2$ \\
\hline$+\mathrm{dP} / \mathrm{dt}$ & $885 \pm 94$ & $+247 \pm 69 * 8$ & $888 \pm 99$ & $-16 \pm 10$ & $+282 \pm 83^{\S}$ \\
\hline $\mathrm{T}_{\mathrm{L}}$ & $56 \pm 6$ & $-10 \pm 4 *$ & $56 \pm 5$ & $-1 \pm 1$ & $-7 \pm 1^{*}$ \\
\hline $\mathrm{T}_{1 / 2}$ & $38 \pm 2$ & $-7 \pm 2 *$ & $38 \pm 2$ & $-2 \pm 1$ & $-6 \pm 2 *$ \\
\hline
\end{tabular}

Atr, Atropine. See Tables I and II for remainder of abbreviations. $* P<0.05$ for within group responses, ${ }^{\S} P<0.05$ versus response in the normal ventricular function group, ${ }^{\ddagger} P<0.05$ for comparison of dobutamine plus acetylcholine response versus dobutamine response.

nation of dobutamine and acetylcholine did not cause any hemodynamic changes in either group.

Dobutamine increased $+\mathrm{dP} / \mathrm{dt}$ in both groups (Table II, Fig. 1), although the inotropic response was significantly greater in the normal ventricular function group. Acetylcholine alone did not change $+\mathrm{dP} / \mathrm{dt}$ in either group. In both groups the combination of dobutamine and acetylcholine was associated with a significant increase in $+\mathrm{dP} / \mathrm{dt}$, although the inotropic response to this combination was significantly less than the response to dobutamine alone. In the normal group acetylcholine resulted in a $60 \pm 8 \%$ inhibition of the inotropic response to dobutamine. Similarly, in the heart failure group acetylcholine caused a $70 \pm 13 \%$ inhibition of the inotropic response to dobutamine.

Dobutamine accelerated left ventricular isovolumic relaxation in both groups (Table II, Fig. 1), consistent with our previous report (24). Similar to its effect on dobutamine-mediated inotropic responses, acetylcholine attenuated the dobutaminemediated reduction in Tau in both groups. Acetylcholine alone had no effect on Tau in the normal group. In contrast, in the heart failure group acetylcholine alone significantly slowed ventricular relaxation. The prolongation of Tau in the heart failure group in response to acetylcholine was significantly different from the response in the normal group.

Protocol 2. Intracoronary dobutamine and atropine. In the normal ventricular function group there was a significant fall in left ventricular end-diastolic pressure in response to both dobutamine and the combination of dobutamine and atropine (Table III). The combination of dobutamine and atropine also increased heart rate in the normal group. There were no hemodynamic changes in the heart failure group.

In the normal ventricular function group the addition of atropine significantly augmented the inotropic response to dobutamine (Table III, Fig. 2), consistent with our previous report (9). The combined infusion of atropine and dobutamine resulted in a $35 \pm 10 \%$ greater increase in $+\mathrm{dP} / \mathrm{dt}$ as compared to dobutamine alone. In contrast, in the heart failure group the addition of atropine did not significantly enhance the inotropic response to dobutamine $(12 \pm 15 \%)$.
A

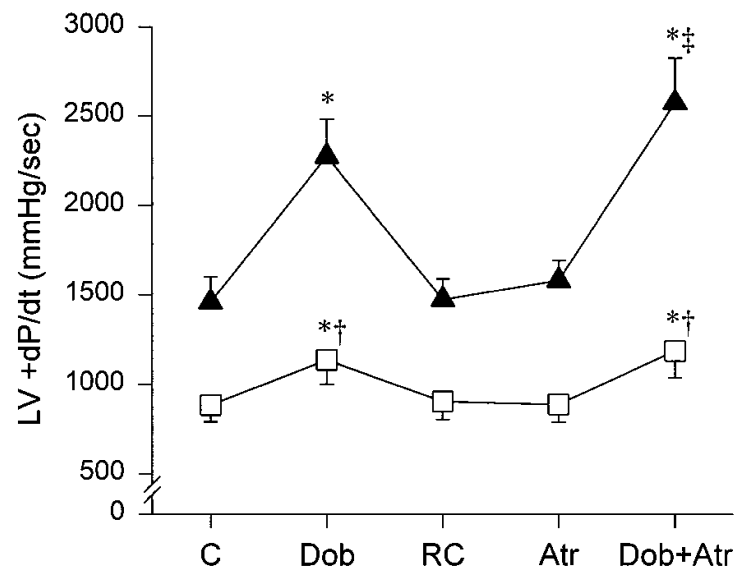

B

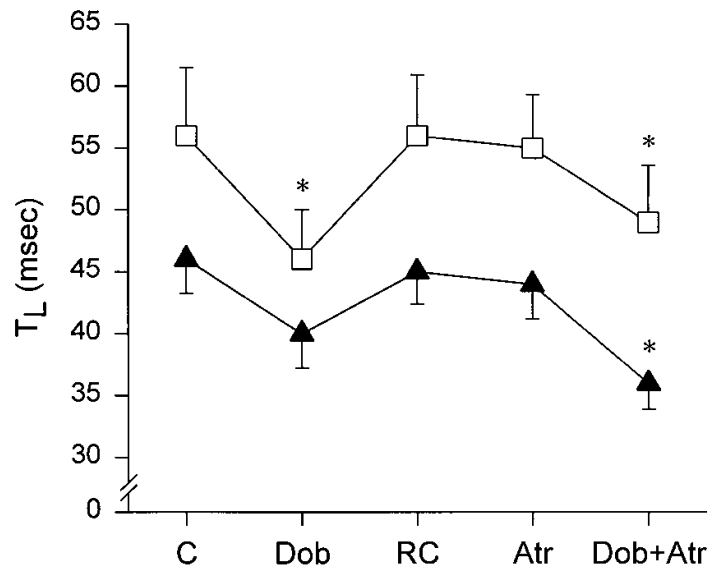

Figure 2. Left ventricular $+\mathrm{dP} / \mathrm{dt}(A)$ and $\mathrm{T}_{\mathrm{L}}(B)$ in the normal ventricular function group (solid triangles) and the heart failure group (open squares) in Protocol 2. Control $(C)$, intracoronary dobutamine $(D o b)$, recontrol $(R C)$, atropine $(A t r)$. Data are presented as mean \pm SEM. $* P<0.05$ versus the preceding control period, ${ }^{\dagger} P<$ 0.05 versus the normal ventricular function group, ${ }^{\ddagger} P<0.05$ for the comparison of dobutamine plus atropine versus dobutamine alone. 
Table IV. Responses to Intravenous Dobutamine, Intracoronary Acetylcholine and Atropine, and Their Combination in Patients with Heart Failure (Protocol 3)

\begin{tabular}{|c|c|c|c|c|c|c|c|}
\hline & $\mathrm{C}$ & $\Delta$ Dob (i.v.) & $\mathrm{RC}$ & $\Delta$ Ach & $\Delta \mathrm{Dob}+\mathrm{Ach}$ & $\Delta \mathrm{Dob}+\mathrm{Ach}+\mathrm{Atr}$ & $\Delta$ Ach + Atr \\
\hline $\mathrm{HR}$ & $86 \pm 8$ & $+10 \pm 6$ & $82 \pm 8$ & $+1 \pm 1$ & $+6 \pm 2$ & $+12 \pm 6^{*}$ & $+7 \pm 8$ \\
\hline LVEDP & $18 \pm 4$ & $-4 \pm 1^{*}$ & $18 \pm 4$ & $+2 \pm 1$ & $-1 \pm 1$ & $-4 \pm 1^{*}$ & $-3 \pm 2$ \\
\hline LVSP & $110 \pm 6$ & $+16 \pm 8^{*}$ & $114 \pm 8$ & $+2 \pm 4$ & $+15 \pm 5^{*}$ & $+19 \pm 6^{*}$ & $-3 \pm 6$ \\
\hline$+\mathrm{dP} / \mathrm{dt}$ & $657 \pm 79$ & $+380 \pm 131 *$ & $685 \pm 82$ & $-21 \pm 8$ & $+179 \pm 69$ & $+390 \pm 128^{*}$ & $+14 \pm 14$ \\
\hline $\mathrm{T}_{\mathrm{L}}$ & $61 \pm 7$ & $-12 \pm 3^{*}$ & $58 \pm 8$ & $+9 \pm 2 *$ & $-1 \pm 41$ & $-9 \pm 3^{*}$ & $0 \pm 1$ \\
\hline $\mathrm{T}_{1 / 2}$ & $46 \pm 4$ & $-9 \pm 2 *$ & $45 \pm 4$ & $+4 \pm 2$ & $-3 \pm 1$ & $-7 \pm 2 *$ & $-3 \pm 4$ \\
\hline
\end{tabular}

$* P<0.05$ for within group comparisons. See Tables I-III for remainder of abbreviations.

Intracoronary dobutamine caused a reduction in Tau which was of borderline statistical significance in the normal ventricular function group and achieved significance in the heart failure group (Table III, Fig. 2). Atropine alone did not change Tau in either group. With the combination of dobutamine and atropine the increase in the rate of isovolumic relaxation in the normal group was now highly significant. In the heart failure group atropine caused no change in the isovolumic relaxation response to dobutamine.

Protocol 3. Intravenous dobutamine and intracoronary atropine and acetylcholine. Six patients with heart failure were studied in Protocol 3. In these patients intravenous dobutamine, alone and combined with intracoronary acetylcholine and atropine, significantly reduced left ventricular end-diastolic pressure and increased left ventricular systolic pressure. The combination of intravenous dobutamine with intracoronary acetylcholine and atropine also caused a significant increase in heart rate. There were no hemodynamic changes in response to intracoronary acetylcholine alone, or combined with atropine.
Intravenous dobutamine increased $+\mathrm{dP} / \mathrm{dt}$ and accelerated isovolumic relaxation (Table IV, Fig. 3). The effect of the combination of intracoronary acetylcholine and intravenous dobutamine was consistent with the results of Protocol 1. In this case acetylcholine caused a $52 \pm 11 \%$ inhibition of the inotropic response to dobutamine and there were no longer significant changes in $+\mathrm{dP} / \mathrm{dt}$, or Tau. The addition of intracoronary atropine to dobutamine and acetylcholine completely restored both the $+\mathrm{dP} / \mathrm{dt}$ and Tau responses to dobutamine, demonstrating that the acetylcholine-mediated suppression of dobutamine responses resulted from stimulation of ventricular muscarinic receptors. Although atropine restored the effects of dobutamine in the presence of acetylcholine, the combination of dobutamine and atropine was not different from the effects of dobutamine alone. The $+\mathrm{dP} / \mathrm{dt}$ response to the combination of intravenous dobutamine and intracoronary atropine and acetylcholine was only $4 \pm 12 \%$ larger than the $+\mathrm{dP} / \mathrm{dt}$ response seen with dobutamine alone.

In Protocol 3 intracoronary acetylcholine slowed left ventricular isovolumic relaxation (Table IV, Fig. 3). Importantly,
A

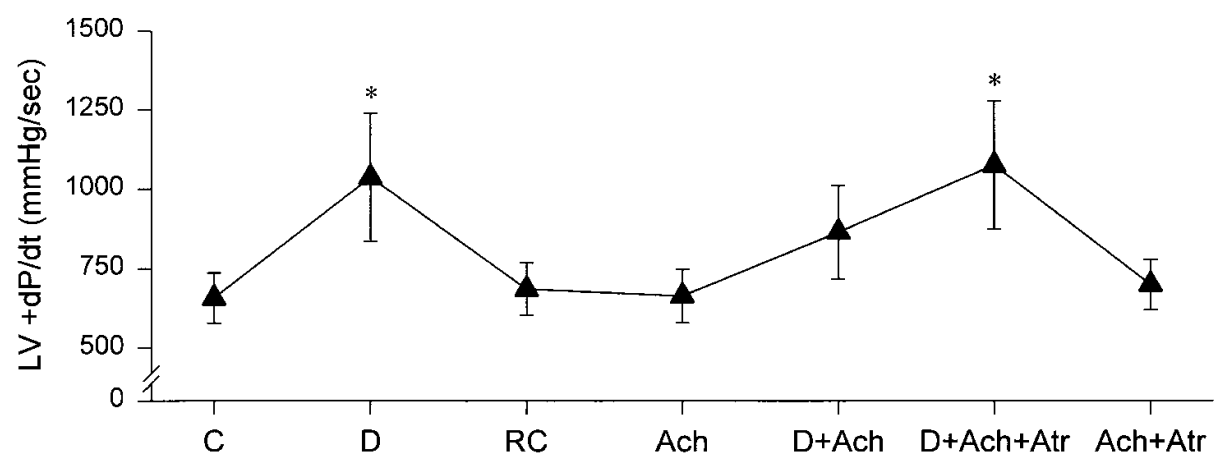

B

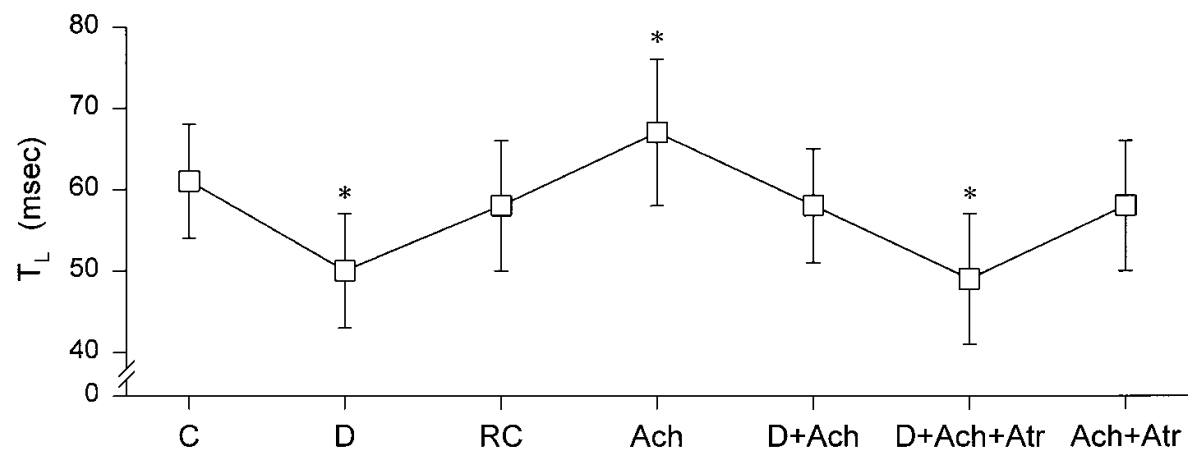

Figure 3. Left ventricular $+\mathrm{dP} /$ $\mathrm{dt}(A)$ and $\mathrm{T}_{\mathrm{L}}(B)$ in the heart failure group studied in Protocol 3. Control $(C)$, intravenous dobutamine $(D)$, recontrol $(R C)$, acetylcholine (Ach), atropine $($ Atr $)$. Data are presented as mean \pm SEM. $* P<0.05$ versus the preceding control period. 
the addition of atropine to acetylcholine completely abolished the prolongation in Tau seen with acetylcholine. This confirms the similar observation in Protocol 1 of a negative lusitropic effect of intracoronary acetylcholine in the heart failure group, and demonstrates that this effect resulted from stimulation of ventricular muscarinic receptors.

\section{Discussion}

The results of this study demonstrate that modulation of cardiac muscarinic receptors affects both basal and $\beta$-adrenergic stimulated left ventricular function in patients with heart failure. The data demonstrate that muscarinic stimulation attenuates $\beta$-adrenergic responses in patients with heart failure, a response similar to that observed in subjects with normal ventricular function. Muscarinic stimulation also slows left ventricular isovolumic relaxation in heart failure patients, an effect not seen in subjects with normal ventricular function. Furthermore, in patients with heart failure these responses to acetylcholine were shown to be mediated by muscarinic receptors since they were completely inhibited by the addition of atropine. Finally, muscarinic blockade with atropine enhanced $\beta$-adrenergic responses in patients with normal ventricular function, a response not observed in those with heart failure.

Intracoronary acetylcholine attenuated the dobutaminemediated inotropic and lusitropic responses in subjects with normal ventricular function and patients with heart failure. The mechanism of this effect of acetylcholine is uncertain, although several possibilities have been suggested. Muscarinic stimulation may have activated the inhibitory $G$ protein, $G_{i}$, leading to reduced adenylate cyclase production of cAMP (25). This may be relevant in the setting of heart failure, where $\mathrm{G}_{\mathrm{i}}$ activity is increased $(5,15-18)$. The effects of acetylcholine may have been secondary to stimulation of inhibitory muscarinic receptors on adrenergic nerve terminals $(6,26)$. This mechanism may be particularly relevant in heart failure, since prejunctional modulation of norepinephrine release is most pronounced in the setting of increased sympathetic nerve firing rate (26), as occurs in heart failure (27). Another potential mechanism relates to cardiac production of cGMP in response to muscarinic stimulation, possibly related to increased nitric oxide activity (8). Increases in cyclic GMP, resulting from muscarinic stimulation, may counteract cAMP mediated increases in contractility (28). In support of this mechanism, Hare et al. demonstrated that nitric oxide mediates part of the muscarinic attenuation of $\beta$-adrenergic mediated increases in contractility in dogs (8).

Intracoronary acetylcholine had a direct negative lusitropic effect in patients with heart failure but not in those with normal ventricular function. In animal models vagal stimulation has been shown to attenuate the increase in $-\mathrm{dP} / \mathrm{dt}$ seen with sympathetic nerve stimulation (7) and to either slow (19), or have no effect (20) on isovolumic relaxation. In patients with heart failure, acetylcholine may have slowed relaxation due to accentuated antagonism (29), a phenomenon whereby the inhibitory effects of muscarinic stimulation are greater as sympathetic activity is increased. Thus, a negative lusitropic effect may have only occurred in those with heart failure because of increased underlying sympathetic activity (27). Alternatively, the negative lusitropic response to acetylcholine may be explained by increased sensitivity to muscarinic stimulation in the setting of heart failure (5). This might occur because of in- creased muscarinic receptor density $(5,12)$, or because of an increase in $G_{i}$ activity in the setting of heart failure $(5,15-18)$. The negative lusitropic effect of acetylcholine may also have resulted from non cAMP-dependent mechanisms including effects on phospholamban phosphorylation (30), and phospholipase C (13), which could stress an already impaired calcium handling system in heart failure (31). Nitric oxide, produced by muscarinic stimulation, may also effect diastolic function. In a recent human study (32), intracoronary nitroprusside, a nitric oxide donor, had no effect on Tau. In contrast, we observed a prolongation of Tau, suggesting that acetylcholine had effects independent of the nitric oxide pathway.

Previous studies have shown that muscarinic receptor density in myocardium from patients with end-stage heart failure is unchanged as compared to receptor density in normal myocardium (13-15), but that $\mathrm{G}_{\mathrm{i}}$ activity is increased (15-17). The results of the present study provide insight into the function of muscarinic pathways in the setting of human congestive heart failure. The suppression of $\beta$-adrenergic responses by acetylcholine in the heart failure group suggests that muscarinic function is intact in the failing human left ventricle. Furthermore, the finding that acetylcholine resulted in a negative lusitropic effect only in the heart failure group suggests that muscarinic responses may actually be increased in the setting of human heart failure, as has recently been demonstrated in an animal model of heart failure (5).

Intracoronary atropine did not augment $\beta$-adrenergic responses in patients with heart failure. There are several possible explanations for this observation. First, the intracoronary concentration of atropine may have been insufficient to antagonize the stimulation of muscarinic receptors by endogenous acetylcholine. However, in the normal ventricular function group the same infusion of atropine augmented dobutamine responses, and the concentration of acetylcholine within ventricular myocardium is likely to have been higher in this group as compared to the heart failure group $(1,2)$. Furthermore, atropine was able to antagonize the effects of exogenous acetylcholine in heart failure patients studied in Protocol 3. Second, the blunted atropine effect may have occurred if muscarinic pathway function is impaired in the setting of heart failure. As discussed above, the results of this study suggest the opposite; the response to muscarinic stimulation is intact, and possibly enhanced in the setting of heart failure. Third, dobutamine may have exhausted the contractile reserve in the heart failure group, thus allowing for no further increase in $+\mathrm{dP} / \mathrm{dt}$ in response to the addition of atropine. In the present study dobutamine was titrated to achieve a $25 \%$ increase in $+\mathrm{dP} / \mathrm{dt}$. This is well below the maximal increase in contractility that has been observed in patients with severe heart failure in response to either intracoronary dobutamine $(56 \%)$, or the combination of intracoronary dobutamine and milrinone (74\%) (33). We hypothesize that the explanation for the lack of an atropine effect in the heart failure group is that there was little endogenous acetylcholine present within ventricular myocardium to antagonize, similar to our previous observation in patients post heart transplantation (9). This hypothesis is consistent with previous investigations that demonstrated reduced parasympathetic tone in heart failure based on heart rate and heart rate variability measurements $(1,2)$.

The methodology used in these experiments has limitations that should be considered. Isovolumic relaxation was measured by the logarithmic method of Weiss et al., $\mathrm{T}_{\mathrm{L}}$ (21) and 
the pressure half-time method of Mirsky, $\mathrm{T}_{1 / 2}$ (22). Although there is no consensus as to the best mathematical estimate of the rate of isovolumic left ventricular relaxation, the methods used in this study have been advocated as reasonable indices of isovolumic relaxation $(21,34,35)$. Despite reports that Tau is sensitive to changes in loading conditions (36), in the human left ventricle Tau has been shown to be insensitive to relatively small changes in load $(34,35)$. Furthermore, changes in loading conditions in the present investigation cannot explain our findings. Dobutamine increased left ventricular systolic pressure (Protocols 1 and 3) which would not explain the observed decreases in Tau. Acetylcholine did not alter left ventricular systolic pressure (Protocols 1 and 3 ) and caused only a small increase in left ventricular end-diastolic pressure (Protocol 1), so changes in loading conditions do not explain the acetylcholine-mediated increase in Tau observed in the heart failure group. The measure of contractility used in these experiments, $+\mathrm{dP} / \mathrm{dt}$, can be influenced by changes in heart rate and loading conditions. Although there were changes in heart rate and loading conditions during some drug infusions these changes were relatively modest and do not explain the observed changes in $+\mathrm{dP} / \mathrm{dt}$. In human experimentation it has been demonstrated that left ventricular $+\mathrm{dP} / \mathrm{dt}$ is quite insensitive to changes in both preload and afterload $(35,37)$. Furthermore, although $+\mathrm{dP} / \mathrm{dt}$ may be effected by changes in heart rate (38), these changes are much larger than the heart rate responses observed in the present experiments. A final limitation is the possible effect of increased coronary blood flow associated with intracoronary acetylcholine. By increasing coronary flow, acetylcholine may reduce the intracoronary concentration of dobutamine, thereby attenuating any dobutamine effect. This mechanism does not explain our findings: in Protocol 3 dobutamine was administered intravenously, so changes in coronary flow would not alter the intracoronary concentration of dobutamine. Importantly, acetylcholine had similar inhibitory effects on dobutamine responses, whether dobutamine was administered via the intracoronary or the intravenous route.

In summary, we have shown that muscarinic stimulation has an independent negative lusitropic effect and antagonizes the effects of $\beta$-adrenergic stimulation in the failing human left ventricle while muscarinic blockade has lesser effects on $\beta$-adrenergic responses in patients with heart failure. The results of this study demonstrate that the responsiveness of muscarinic receptors is intact, and possibly enhanced in the setting of heart failure. A potentially important implication of this study is that augmentation of parasympathetic outflow in patients with heart failure may have important effects on left ventricular function. This should be recognized during future evaluation of parasympathomimetic agents for the treatment of congestive heart failure.

\section{Acknowledgments}

J.D. Parker is a scholar of the Medical Research Council of Canada. This work was supported in part by funding from the Heart and Stroke Foundation of Ontario (no. A2220), Bayer Inc., and grant HL52320 from the National Institutes of Health.

\section{References}

1. Eckberg, D.L., M. Drabinsky, and E. Braunwald. 1971. Defective cardiac parasympathetic control in patients with heart disease. N. Engl. J. Med. 285:
877-883.

2. Porter, T.R., D.L. Eckberg, J.M. Fritsch, R.F. Rea, L.A. Beightol, J.F Schmedtje, and P.K. Mohanty. 1990. Autonomic pathophysiology in heart failure patients. Sympathetic-cholinergic interrelations. J. Clin. Invest. 85:13621371 .

3. De Ferrari, G.M., E. Vanoli, P. Curcuruto, G. Tommasini, and P.J. Schwartz. 1992. Prevention of life-threatening arrhythmias by pharmacologic stimulation of the muscarinic receptors with oxotremorine. Am. Heart. J. 124: 883-890.

4. La Rovere, M.T., A. Mortara, P. Pantaleo, R. Maestri, F. Cobelli, and L. Tavazzi. 1994. Scopolamine improves autonomic balance in advanced congestive heart failure. Circulation. 90:838-843.

5. Vatner, D.E., N. Sato, J.B. Galper, and S.F. Vatner. 1996. Physiological and biochemical evidence for coordinate increases in muscarinic receptors and $\mathrm{G}_{\mathrm{i}}$ during pacing induced heart failure. Circulation. 94:102-107.

6. Levy, M.N., and B. Blattberg. 1976. Effect of vagal stimulation on the overflow of norepinephrine into the coronary sinus during cardiac sympathetic nerve stimulation in the dog. Circ. Res. 38:81-85.

7. Henning, R.J., I.R. Khalil, and M.N. Levy. 1990. Vagal stimulation attenuates sympathetic enhancement of left ventricular function. Am. J. Physiol. 258: H1470-H1475.

8. Hare, J.M., J.F. Keaney, J.L. Balligand, J. Loscalzo, T.W. Smith, and W.S. Colucci. 1995. Role of nitric oxide in parasympathetic modulation of $\beta$-adrenergic myocardial contractility in normal dogs. J. Clin. Invest. 95:360-366.

9. Landzberg, J.S., J.D. Parker, D.F. Gauthier, and W.S. Colucci. 1994. Effect of intracoronary acetylcholine and atropine on basal and dobutamine-stimulated left ventricular contractility. Circulation. 89:164-168.

10. Vatner, D.E., D.L. Lee, K.R. Schwarz, J.P. Longabaugh, A.M. Fujii, S.F. Vatner, and C.J. Homcy. 1988. Impaired cardiac muscarinic receptor function in dogs with heart failure. J. Clin. Invest. 81:1836-1842.

11. Gopalakrishnan, M., D.J. Triggle, A. Rutledge, Y.W. Kwon, J.A. Bauer, and H.L. Fung. 1991. Regulation of $\mathrm{K}^{+}$and $\mathrm{Ca}^{2+}$ channels in experimental cardiac failure. Am. J. Physiol. 261:H1979-H1987.

12. Wilkinson, M., A. Giles, J.A. Armour, and R. Cardinal. 1996. Ventricular, but not atrial, $\mathbf{M}_{2}$-muscarinic receptors increase in the canine pacing-overdrive model of heart failure. Can. J. Cardiol. 11:71-76.

13. Bristow, M.R. 1993. Changes in myocardial and vascular receptors in heart failure. J. Am. Coll. Cardiol. 22:61A-71A.

14. Bohm, M., M. Ungerer, and E. Erdmann. 1990. Beta adrenoceptors and $\mathrm{m}$-cholinoceptors in myocardium of hearts with coronary artery disease or idiopathic dilated cardiomyopathy removed at cardiac transplantation. Am. J. Cardiol. 66:880-882.

15. Bohm, M., P. Gierschik, K. Jakobs, B. Pieske, P. Schnabel, M. Ungerer, and E. Erdmann. 1990. Increase of $\mathrm{G}_{\mathrm{ia}}$ in human hearts with dilated but not ischemic cardiomyopathy. Circulation. 82:1249-1265.

16. Feldman, A.M., A.E. Cates, W.B. Veazey, R.E. Hershberger, M.R Bristow, K.L. Baughman, W.A. Baumgartner, and C. Van Dop. 1988. Increase of the $40,000-\mathrm{mol}$ wt pertussis toxin substrate ( $\mathrm{G}$ protein) in the failing human heart. J. Clin. Invest. 82:189-197.

17. Hershberger, R.E., A.M. Feldman, and M.R. Bristow. 1991. A -Adenosine receptor inhibition of adenylate cyclase activity in failing and nonfailing human ventricular myocardium. Circulation. 83:1343-1351.

18. Eschenhagen, T., U. Mende, M. Nose, W. Schmitz, H. Scholz, A. Haverich, S. Hirt, V. Doring, P. Kalmar, P. Hoppner, and H. Seitz. 1992. Increased messanger RNA level of the inhibitory $\mathrm{G}$ protein a subunit $\mathrm{G}_{\mathrm{ia}}{ }^{-2}$ in human endstage heart failure. Circ. Res. 70:688-696.

19. Henning, R.J., J. Cheng, and M.N. Levy. 1989. Vagal stimulation decreases rate of left ventricular relaxation. Am. J. Physiol. 256:H428-H433.

20. Xenopoulos, N.P., and R.J. Applegate. 1994. The effect of vagal stimulation on left ventricular systolic and diastolic performance. Am. J. Physiol. 266: H2167-H2173.

21. Weiss, J.L., J.W. Frederiksen, and M.L. Weisfeldt. 1976. Hemodynamic determinants of the time-course of fall in canine left ventricular pressure. $J$. Clin. Invest. 58:751-760.

22. Mirsky, I. 1984. Assessment of diastolic function: suggested methods and future considerations. Circulation. 69:836-841.

23. Floras, J.S. 1993. Clinical aspects of sympathetic activation and parasympathetic withdrawal in heart failure. J. Am. Coll. Cardiol. 22:72A-84A.

24. Parker, J.D., J.S. Landzberg, J.A. Bittl, I. Mirsky, and W.S. Colucci. 1991. Effects of $\beta$-adrenergic stimulation with dobutamine on isovolumic relaxation in the normal and failing left ventricle. Circulation. 84:1040-1048.

25. Fleming, J.W., R.A. Strawbridge, and A.M. Watanabe. 1987. Muscarinic receptor regulation of cardiac adenylate cyclase activity. J. Mol. Cell. Cardiol. 19:47-61.

26. Muscholl, E. 1980. Peripheral muscarinic control of norepinephrine release in the cardiovascular system. Am. J. Physiol. 239:H713-H720.

27. Meredith, I.T., G. Eisenhofer, G.W. Lambert, E.M. Dewar, G.L. Jennings, and M.D. Esler. 1993. Cardiac sympathetic nervous activity in congestive heart failure: evidence for increased neuronal norepinephrine release and preserved neuronal uptake. Circulation. 88:136-145.

28. Hartzell, H.C., and R. Fischmeister. 1986. Opposite effects of cyclic AMP and cyclic GMP on $\mathrm{Ca}^{2+}$ current in single heart cells. Circ. Res. 29:437-445.

29. Levy, M.N. 1971. Sympathetic-parasympathetic interactions in the 
heart. Circ. Res. 29:437-445.

30. Gupta, R.C., J. Neumann, and A.M. Watanabe. 1993. Comparison of adenosine and muscarinic receptor-mediated effects on protein phosphatase inhibitor-1 activity in the heart. J. Pharmacol. Exp. Ther. 266:16-22.

31. Arai, M., N.R. Alpert, D.H. Maclennan, P. Barton, and M. Periasamy. 1993. Alterations in sarcoplasmic reticulum gene expression in human heart failure: a possible mechanism for alterations in systolic and diastolic properties of the failing myocardium. Circ. Res. 72:463-469.

32. Paulus, W.J., P.J. Vantrimpont, and A.M. Shah. 1994. Acute effects of nitric oxide on left ventricular relaxation and diastolic distensibility in humans. Circulation. 89:2070-2078.

33. Colucci, W.S., A.R. Denniss, G.F. Leatherman, R.J. Quigg, P.J. Ludmer, J.D. Marsh, and D.F. Gauthier. 1988. Intracoronary infusion of dobutamine to patients with and without severe congestive heart failure. J. Clin. Invest. 81:1103-1110.

34. Varma, S.K., R.M. Owen, M.L. Smucker, and M.D. Feldman. 1989. Is $t$ a preload-independent measure of isovolumetric relaxation? Circulation. 80: 1757-1765.

35. Starling, M.R., D.G. Montgomery, G.B.J. Mancini, and R.A. Walsh. 1987. Load independence of the rate of isovolumic relaxation in man. Circulation. 76:1274-1281.

36. Blaustein, A.S., and W.H. Gaasch. 1983. Myocardial relaxation. VI. Effects of $\beta$-adrenergic tone and asynchrony on LV relaxation rate. Am. J. Physiol. 244:H417-H422.

37. Jaski, B.E., M.A. Fifer, R.F. Wright, E. Braunwald, and W.S. Colucci. 1985. Positive inotropic and vasodilator actions of milrinone in patients with se vere congestive heart failure. Dose-response relationships and comparison to nitroprusside. J. Clin. Invest. 75:643-649.

38. Feldman, M.D., J.D. Alderman, J.M. Aroesty, H.D. Royal, J.J. Ferguson, R.M. Owen, W. Grossman, and R.G. McKay. 1988. Depression of systolic and diastolic myocardial reserve during atrial pacing tachycardia in patients with dilated cardiomyopathy. J. Clin. Invest. 82:1661-1669. 